\title{
P-0454: Resistance exercise ameliorates diabetic cardiomyopathy
}

J.C. Won ${ }^{1}$, Y.C. Hwang'2, S.Y. Kim³ ${ }^{3}$, J. Han' ${ }^{4}$.

${ }^{1}$ Inje University School of Medicine- Sanggye Paik Hospital, Department of Internal Medicine, Seoul, Korea, ${ }^{2}$ Kyung Hee University

Hospital at Gangdong, Department of Endocrinology and Metabolism, Seoul, Korea, ${ }^{3}$ Chosun University Hospital- Chosun

University School of Medicine, epartment of Internal Medicine, Gwangju, Korea, ${ }^{4}$ Cardiovascular and Metabolic Disease Center-

College of Medicine Inje University, Department of Physiology, Busan, Korea.

\section{INTRODUCTION}

Diabetic cardiomyopathy (DC) is a hallmark complication of long-standing hyperglycemia $t$ caused by various metabolic and mitochondrial disturbances. Physical activities such as exercise do not only enhance conditions in healthy individuals but could also improve the state of those afflicted with diseases. However, the beneficial effects of resistance exercise in the prevention of DC and cardiac mitochondrial dysfunction are still uncertain. In this study, we investigated whether RE attenuates DC through improving mitochondrial function.

\section{METHODS}

We utilized an in vivo rat model of diabetes. Fourteen Otsuka Long-Evans Tokushima Fatty rats were assigned to sedentary control (SC, $n=7)$ and RE $(n=7)$ groups at 28 weeks of age. Long-Evans Tokushima Otsuka rats were used as the nondiabetic control. RE were trained by 20 repetitions of climbing a ladder 5 days per week.

\section{RESULTS}
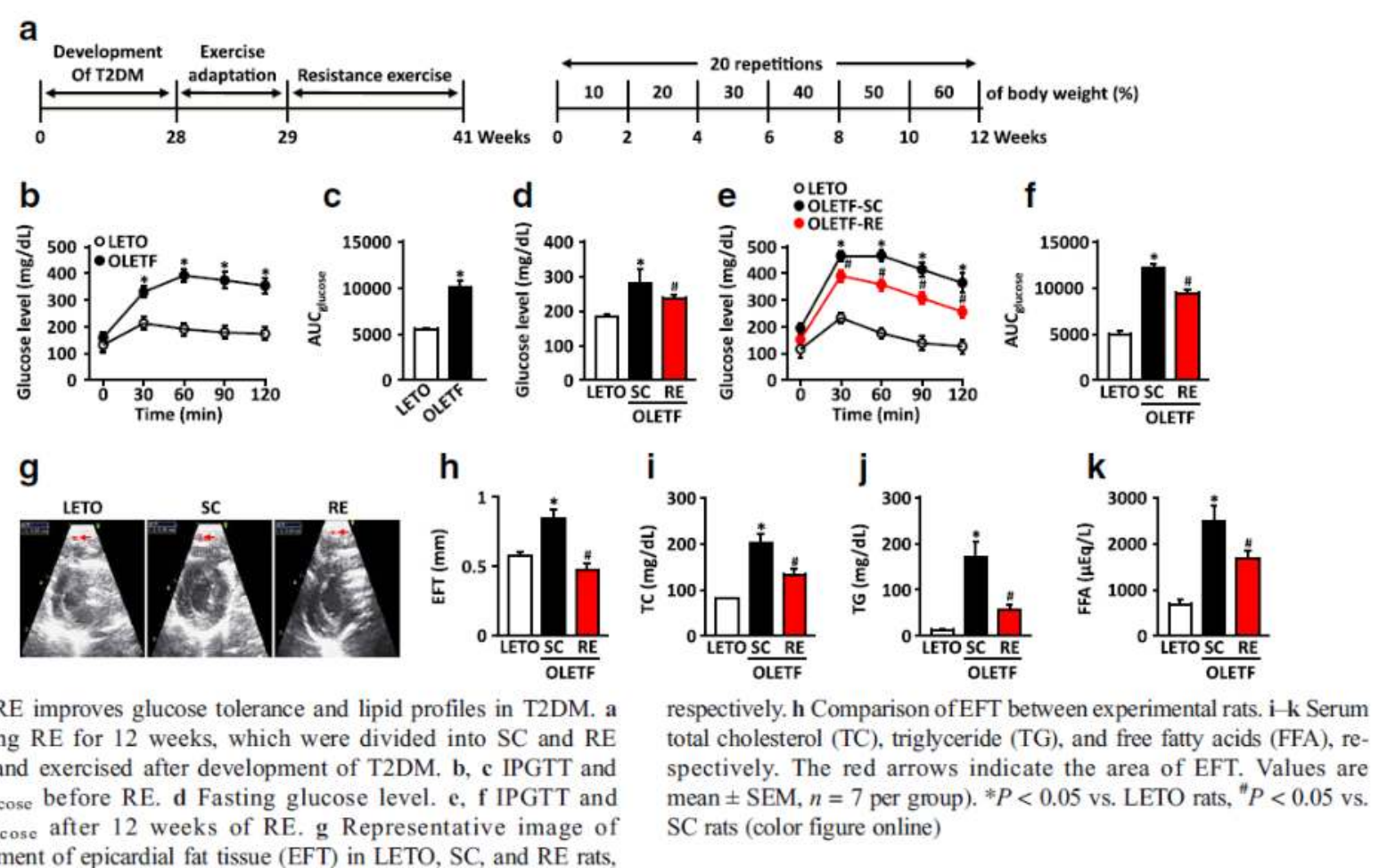

\begin{tabular}{|c|c|c|c|c|}
\hline \multirow{17}{*}{$\begin{array}{l}\text { Table } 1 \text { Body weight, mean } \\
\text { daily food intake, and tissue } \\
\text { weight of experimental rats after } \\
12 \text { weeks of RE }\end{array}$} & & LETO $(n=7)$ & \multirow[t]{2}{*}{$\mathrm{SC}(n=7)$} & \multirow[t]{2}{*}{$\operatorname{RE}(n=7)$} \\
\hline & \multicolumn{2}{|l|}{ Before RE } & & \\
\hline & Body weight (g) & $462.71 \pm 6.23$ & $566.00 \pm 13.85^{*}$ & $567.00 \pm 7.35^{*}$ \\
\hline & Daily food intake (day $/ \mathrm{g})$ & $21.80 \pm 0.82$ & $29.62 \pm 1.40^{*}$ & $26.18 \pm 0.80^{*}$ \\
\hline & \multicolumn{4}{|l|}{ After RE } \\
\hline & Body weight (g) & $518.71 \pm 10.12$ & $571.50 \pm 22.09 *$ & $523.29 \pm 13.34 * *$ \\
\hline & Daily food intake (day/g) & $20.31 \pm 1.05$ & $34.11 \pm 2.20^{*}$ & $28.68 \pm 2.38^{*}$ \\
\hline & HW (g) & $1.33 \pm 0.03$ & $1.52 \pm 0.05^{*}$ & $1.59 \pm 0.06^{*}$ \\
\hline & $\mathrm{HW} / \mathrm{BW}(\mathrm{mg} / \mathrm{g})$ & $2.56 \pm 0.05$ & $2.66 \pm 0.09$ & $3.05 \pm 0.10^{* * * * *}$ \\
\hline & $\mathrm{LV} / \mathrm{BW}(\mathrm{mg} / \mathrm{g})$ & $2.29 \pm 0.20$ & $2.27 \pm 0.06$ & $2.12 \pm 0.04$ \\
\hline & $\mathrm{SW}(\mathrm{g})$ & $0.46 \pm 0.02$ & $0.39 \pm 0.03$ & $0.42 \pm 0.01$ \\
\hline & $\mathrm{SW} / \mathrm{BW}(\mathrm{mg} / \mathrm{g})$ & $0.88 \pm 0.03$ & $0.68 \pm 0.06^{*}$ & $0.81 \pm 0.03^{* *}$ \\
\hline & GW (g) & $4.62 \pm 0.08$ & $4.08 \pm 0.25 *$ & $4.23 \pm 0.12$ \\
\hline & $\mathrm{GW} / \mathrm{BW}(\mathrm{mg} / \mathrm{g})$ & $8.91 \pm 0.13$ & $7.12 \pm 0.29 *$ & $8.10 \pm 0.22^{* * * *}$ \\
\hline & & & & \\
\hline & $\begin{array}{l}\text { LETO Long Evans Tokush } \\
\text { resistant exercise, } H W \text { hea } \\
\text { nemius muscle weight }\end{array}$ & $\begin{array}{l}\text { OLETy ot } \\
W \text { body wei }\end{array}$ & $\begin{array}{l}\text { vans Tokushima } \\
\text { entricle, } S W \text { sol }\end{array}$ & $\begin{array}{l}\text { dentary control, } R E \\
\text { veight, } G W \text { gastroc- }\end{array}$ \\
\hline & ${ }^{*} P<0.05$ vs. LETO rats; & vs. SC rats & & \\
\hline
\end{tabular}
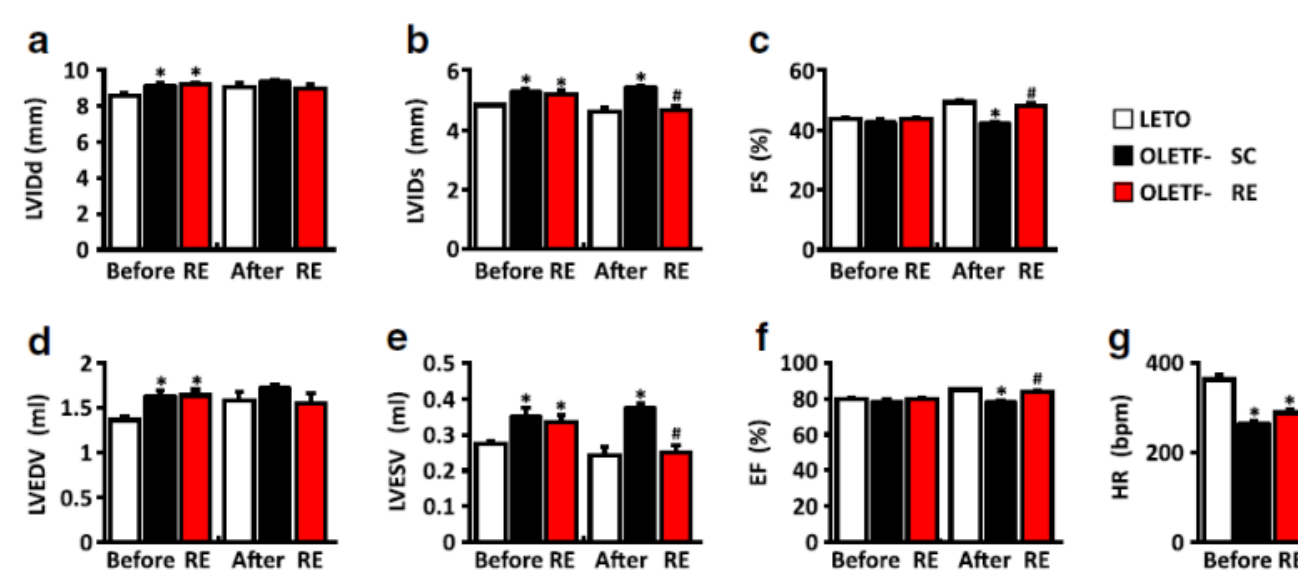

$\begin{array}{ll}\text { Fig. } 2 \text { RE enhances cardiac contraction in T2DM. a-c Comparison of } & \text { (LVEDV) and systole (LVESV) and ejection fraction (EF) between } \\ \text { left ventricular intermal dimension in diastole (LIDd) and systole } & \text { experimental rats, respectively. g Heart rate (HR). Values are }\end{array}$

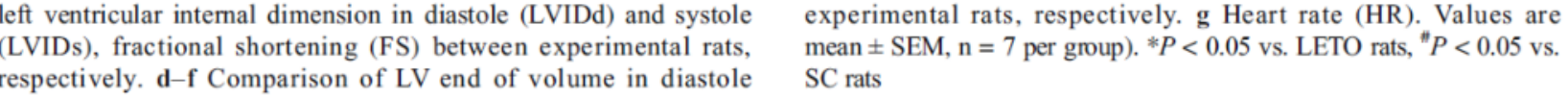

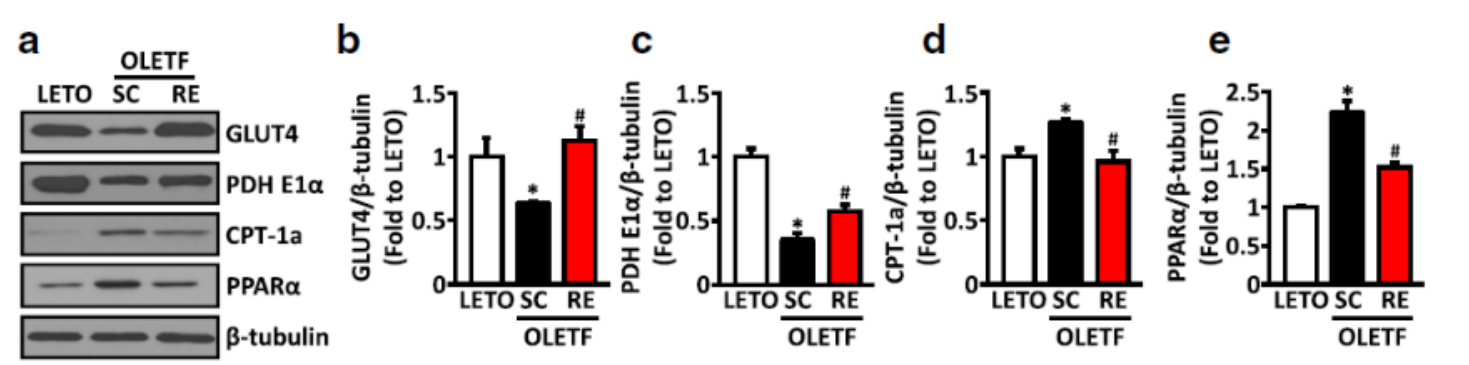

Fig. 3 RE regulates energy metabolism in T2DM hearts. a The E1 $\alpha$, CPT- $1 \alpha$, and PPAR $\alpha$ to $\beta$-tubulin levels were determined by $\begin{array}{ll}\text { expression of proteins related to glucose (GLUT4 and PDH E1 } \alpha \text { ) and } & \begin{array}{l}\text { image J. Vilues are mean } \pm \text { SEM }\left(n=4 \text { per group). }{ }^{*} P<0.05 \text { vs. }\right. \\ \text { LETO rats, } P\end{array} P<0.05 \text { vs. SC rats }\end{array}$ fat (CPT-1 $\alpha$ and PPAR $\alpha$ ) metabolism were evaluated by western
blotting. b-e $\mathrm{e}$ The relative ratio of proteins, including GLUT4, PDH
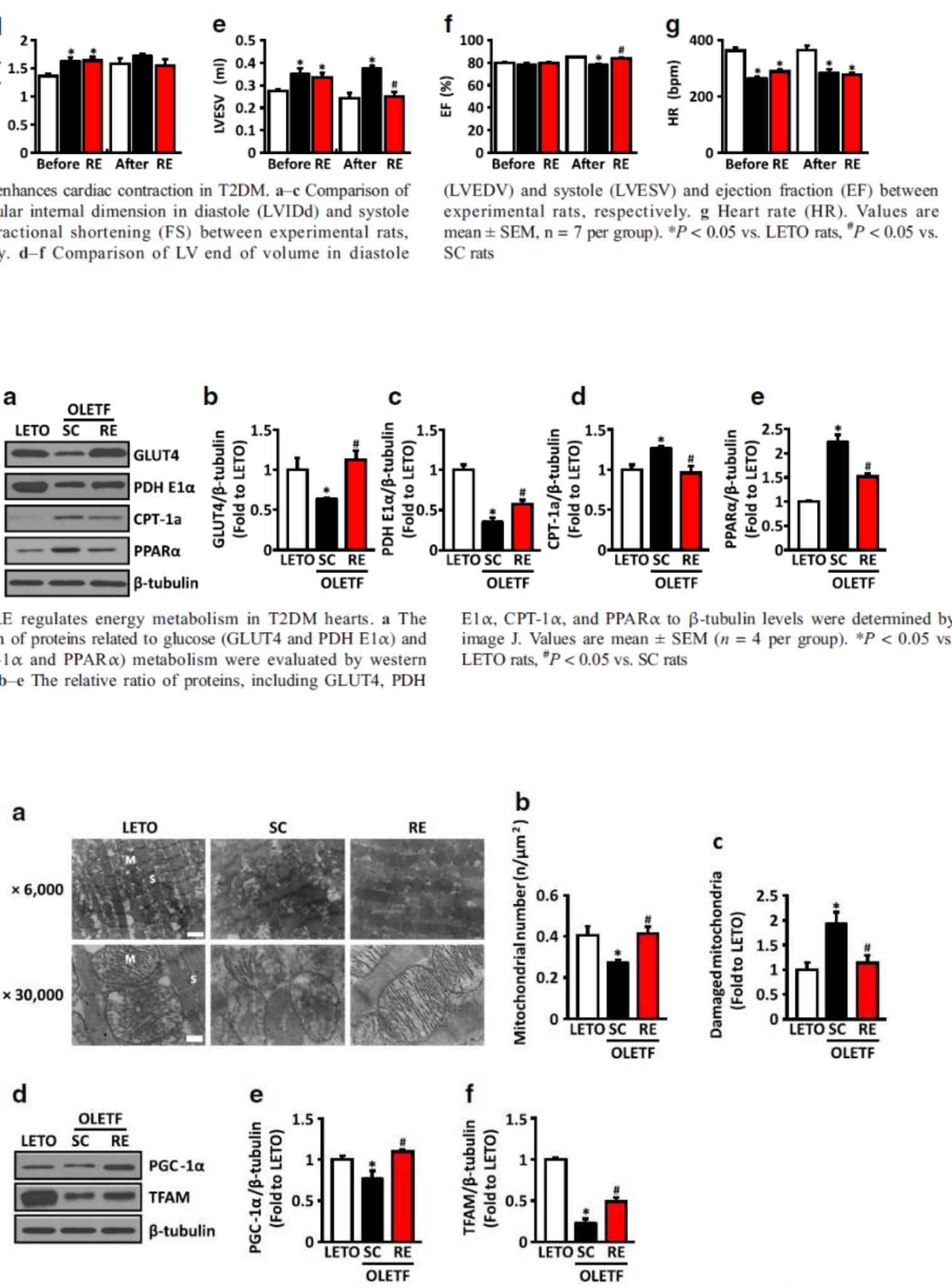

Fig. 4 RE alters mitochondrial morphology in T2DM hearts. a represents as a fold to LETO. $\mathrm{d}-\mathrm{f}$ PGC-1 $\alpha$ and TFAM protein

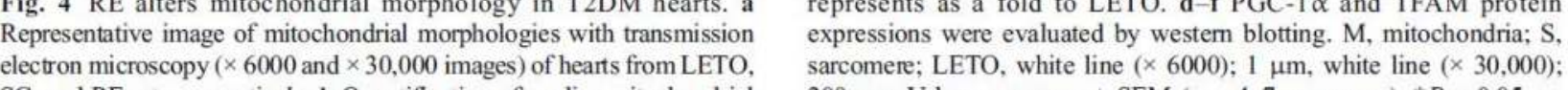

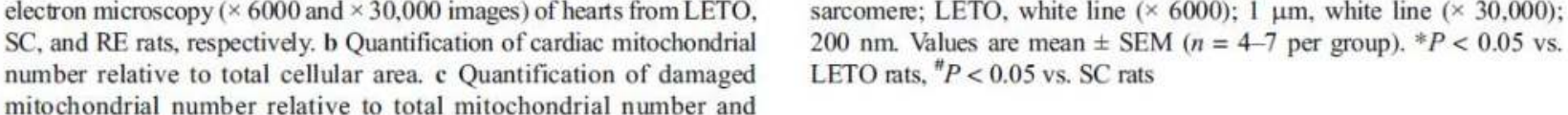

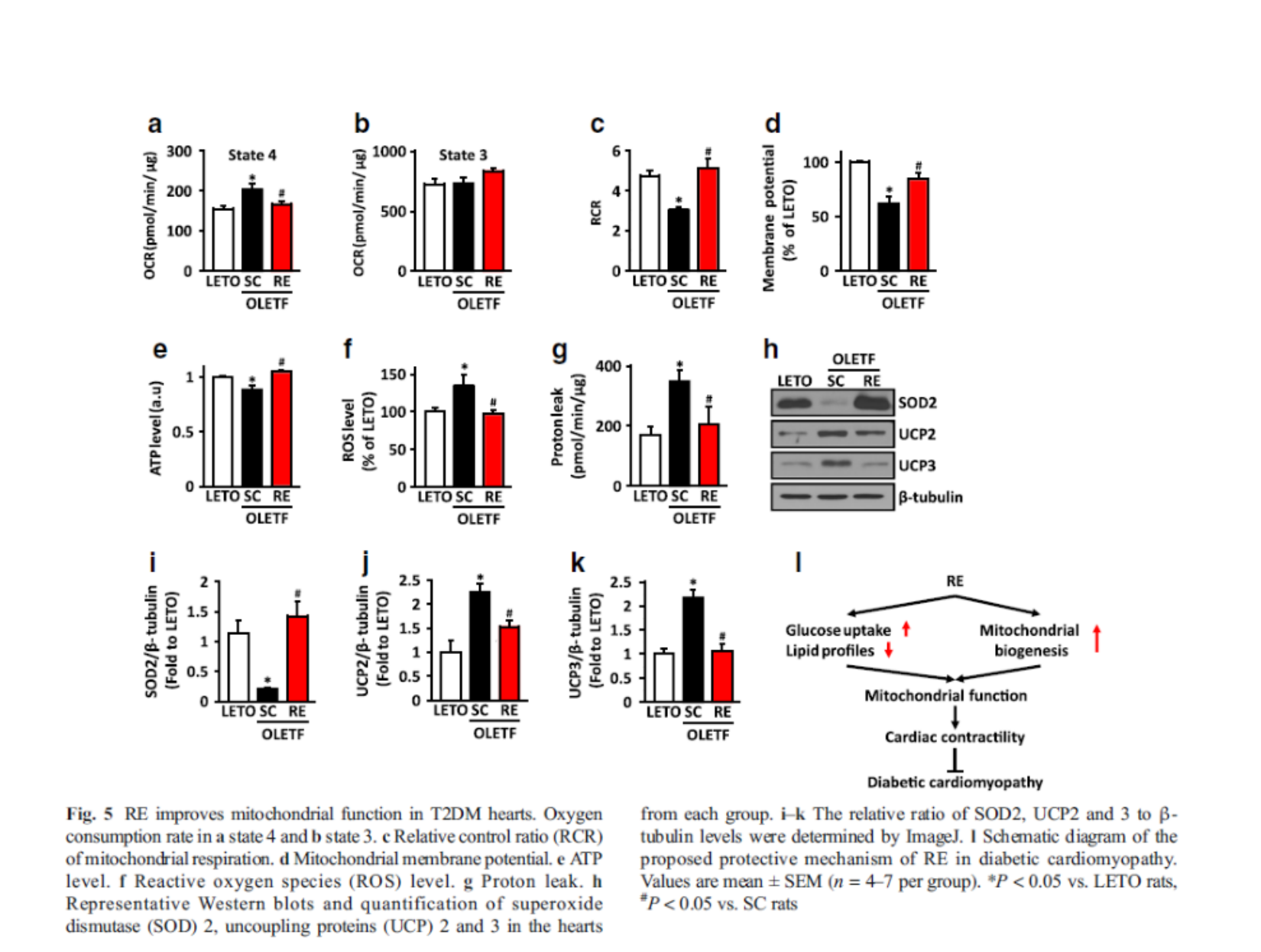

\section{CONCLUSION}

$R E$ is effective in the prevention of cardiac dysfunction along the course of diabetes in relation to attenuation of metabolic disturbances and mitochondrial dysfunction, which may contribute to enhanced contractility of diabetic heart in animal models. 